\title{
Preface to the special issue on high entropy materials and tungsten-based nuclear materials
}

\author{
Chen-Yang $\mathrm{Lu}^{1}{ }^{1} \cdot \mathrm{Y} \cdot \mathrm{i}-\mathrm{Ping} \mathrm{Lu}^{2}$ (D)
}

Published online: 20 May 2021

(C) The Nonferrous Metals Society of China 2021

For the past decade and a half, high entropy alloys have drawn great research interest in both materials science and engineering society. Unlike conventional alloys, which normally contain one or two major elements, high entropy alloys comprise multiple principal elements in high concentrations. The multi-dimensional composition space from this alloying strategy can greatly expand the alloy design range and offer new possibilities for improving material performance. The multiprincipal-element character of high entropy alloys leads to four important core effects, including high entropy effect, sluggish diffusion effect, severe-latticedistortion effect and cocktail effect. The core effects provide novel properties to high entropy alloys, such as excellent specific strength, superior mechanical performance at high temperatures, exceptional ductility and fracture toughness at cryogenic temperatures, superconductivity and excellent radiation resistance. Therefore, high entropy alloys have good potential application prospects in coating materials, low-temperature structural materials, thermoelectric materials, superconducting materials, aerospace materials and nuclear materials. Furthermore, the concept of "high entropy" has now extended from alloys to other functional materials. Recent studies have reported that some high entropy materials possess excellent catalytic and hydrogenabsorbing properties, greatly expanding the application areas of high entropy materials.

One topic of this special issue is about the preliminary researches of high entropy materials. It consists of six review papers from researchers in various countries including

Chen-Yang Lu

chenylu@xjtu.edu.cn

Yi-Ping Lu

luyiping@dlut.edu.cn

1 Department of Nuclear Science and Technology, Xi' an Jiaotong University, Xi' an 710049, China

2 School of Materials Science and Engineering, Dalian University of Technology, Dalian 116024, China
China, Sweden, and Australia. Prof. Yi-Ping Lu reviews the manufacturing and service performance of tungstencontaining high entropy alloys. Dr. Hua-Hai Shen presents the preliminary assessment of high entropy alloys for tritium storage. Dr. Tan Shi reviews the current development of body-centered cubic high entropy alloys, especially refractory high entropy alloys, on its potential use in both fission and fusion reactors. Dr. He Huang specifically focused his review on W-based high entropy alloys that are considered as candidate materials in nuclear fusion. In addition to high entropy metals, an interesting perspective review about high entropy carbide ceramics has been reported by Prof. ZhengGang Wu. Dr. Wen-Yi Huo presents recent progress on high entropy materials used as high-performance catalysts for electrocatalytic water splitting applications.

Interestingly, four of the papers above specifically studied or at least discussed the potential of high entropy alloys for nuclear applications, indicating the urgent needs to develop advanced materials for new-generation nuclear energy. Following this demand, this special topic issue also includes two articles on W-based nuclear materials used in fusion reactors. Dr. Wei Liu reviews the recent progress of the laser powder bed fusion fabrication of tungsten, which is a type of additive manufacturing technology that can easily fabricate complex parts used in fusion reactors. A research article from Prof. Qing Peng investigates the defect evolution in tungsten by molecular dynamics method.

We hope this issue will provide a good inspiration for researchers, scientists and graduate students who have interest in high entropy materials, leading more researchers to explore this limitless and promising field.

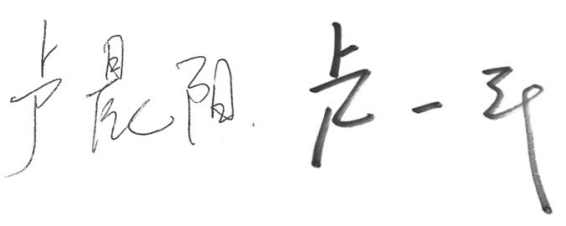



Dr. Chen-Yang Lu
Dr. Yi-Ping Lu
May 01, 2021

Publisher's Note Springer Nature remains neutral with regard to jurisdictional claims in published maps and institutional affiliations.

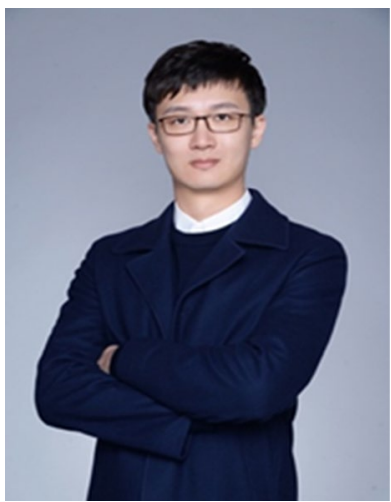

Dr. Chen-Yang Lu is a professor in the Department of Nuclear Science and Technology of Xi'an Jiaotong University, China. He received his Ph.D. in Materials Science from Northeastern University in 2014. He worked at the University of Michigan as a post-doctoral fellow and research scientist in the Department of Nuclear Engineering \& Radiological Sciences from 2014 to 2018. Prof. Lu engaged in the research of nuclear engineering materials. He dedicated his researches on the preparation of advanced nuclear structural materials, radiation damages in materials, and advanced microstructural characterizations. $\mathrm{He}$ has published more than 50 SCI peer-reviewed papers, including 4 Nature Communications, 4 Acta Materialia, 10 Journal of Nuclear Materials and other influential papers in this field. The articles have been cited more than 2,100 times with a h-index of 23 .

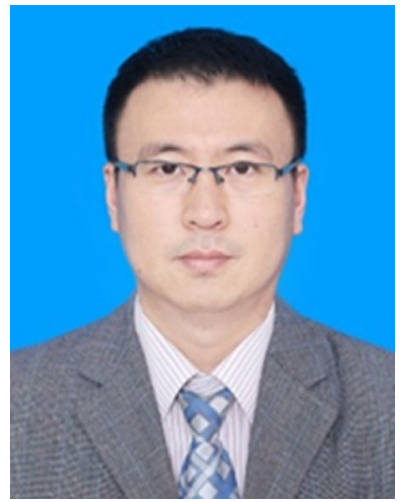

Dr. Yi-Ping Lu is a professor of Materials Science and Engineering at the Dalian University of Technology (DUT), China. Prof. Lu was selected as the Young and Middle-aged Innovation Leaders of the "Ministry of Science and Technology of China", the "Outstanding Youth Fund of the National Natural Science Foundation of China", the Top Youth Talents of Liaoning Province, and the Outstanding Young Scientific and Technological Talents of Dalian City. His research mainly focuses on composition design, smelting, solidified structure control, microstructural analysis and performance test of high-entropy alloys (HEAs). Dr. Lu has published 70 peer-reviewed journal papers on HEAs with a total of over 3000 citations based on the "Google Scholar". 